\title{
The utility of linking National Food Ingredient Databases to National Food Consumption surveys: a pilot study on fibre and sugar
}

\author{
Sarah Kelly ${ }^{1}$, Aoibhinn Moore-Heslin², Mengna Yang ${ }^{2}$, Maria Buffini ${ }^{2}$, Laura Kehoe ${ }^{3}$, \\ John Kearney ${ }^{4}$, Janette Walton ${ }^{5}$, Albert Flynn ${ }^{3}$, Breige McNulty ${ }^{2}$ and Anne Nugent ${ }^{1,2}$ \\ ${ }_{1}^{1}$ Institute for Global Food Security, School of Biological Sciences, Queens University Belfast, Belfast, United Kingdom, \\ ${ }^{2}$ Institute of Food and Health, School of Agriculture and Food Science, University College Dublin, Dublin, Ireland, \\ ${ }^{3}$ School of Food and Nutritional Sciences, University College Cork, Cork, Ireland, \\ ${ }^{4}$ School of Biological \& Health Sciences, Technological University Dublin, Dublin, Ireland and \\ ${ }^{5}$ Department of Biological Sciences, Cork Institute of Technology, Cork, Ireland, Cork, Ireland
}

\begin{abstract}
Introduction: Current dietary recommendations encourage increased fibre and reduced sugar consumption. In the UK, specific targets and benchmarks have been established for the sugar content of some foods but not for fibre. National Food Consumption Surveys provide comprehensive information of all foods consumed by representative population samples. The Irish national food surveys as completed by the Irish Universities Nutrition Alliance (IUNA) capture dietary data at brand level with all details as gathered on pack entered into a discrete but inter-linked database, the Irish National Food Ingredient Database (INFID). The aim of this study was to profile the carbohydrate quality of a convenience sub-sample of packaged foods as eaten by Irish children during the National Children's Food Survey II (2017/2018) as entered into INFID.
\end{abstract}

Materials and Methods: All on-pack details from 385 available foods in the categories 'white breads and rolls'; 'brown breads and rolls'; 'other breads and scones'; 'ready to eat breakfast cereals (RTEBC)'; 'biscuits'; and 'cakes, buns and pastries' were entered in to INFID and quality control completed. The carbohydrate profile of the products was assessed with respect to fibre labelling criteria and UK sugar guidelines and targets. SPSS Version 25 was used for all analyses.

Results: Although 56\% (n210) of all products entered were eligible to make a 'source of' or 'high' fibre claim, only 20\% (n78) made such a claim. Of this, 46\% stated 'high fibre' and 32\% 'source', predominately in the 'brown breads and rolls' and 'RTEBC' groups. When compared to UK Department of Health guidance for 'low', 'medium' and 'high' sugar, 65\% of all products examined (n250) were either 'low' or 'medium' sugar. Comparison of median sugar contents with Public Health England sugar reformulation targets revealed different responses in each category, with all categories other than foods deemed as "morning goods" yet to meet the 2020 target of $20 \%$ reduction in sugar content.

Discussion: This small pilot study of a convenience sample of foods suggests that for the limited number of foods examined, for some there remains challenges to reduce sugar and increase fibre contents. Strategies such as reformulation, change in portion size, flexibility in labelling and/or a shift in sales portfolios could be considered but only alongside technological and safety considerations. Further research to broaden this analysis and to link nutrient levels as listed on pack with actual consumption patterns could help ensure all recent initiatives including reformulation are recognised.

\section{Conflict of Interest}

There is no conflict of interest. 